\title{
Virtual Instrumentation for Study of a Fluid Power System
}

\section{Dr. Alamgir A. Choudhury, Western Michigan University}

Alamgir A. Choudhury is an Associate Professor of Engineering Design, Manufacturing and Management Systems at Western Michigan University, Kalamazoo, Michigan. His MS and PhD are in mechanical engineering from NMSU (Las Cruces) and BS in mechanical engineering from BUET (Dhaka). His interest includes computer applications in curriculum, MCAE, mechanics, fluid power, and instrumentation \& control. He is a Registered Professional Engineer in the State of Ohio and affiliated with ASME, ASEE, SME and TAP.

\section{Dr. Jorge Rodriguez P.E., Western Michigan University}

Professor in the Department of Engineering Design, Manufacturing, and Management Systems (EDMMS) at Western Michigan University's (WMU). Co-Director of the Center for Integrated Design (CID), and currently the college representative to the President's University-wide Sustainability Committee at WMU. Received his Ph.D. in Mechanical Engineering-Design from University of Wisconsin-Madison and received an MBA from Rutgers University. His B.S. degree was in Mechanical and Electrical Engineering at Monterrey Tech (ITESM-Monterrey Campus). Teaches courses in CAD/CAE, Mechanical Design, Finite Element Method and Optimization. His interest are in the area of product development, topology optimization, additive manufacturing, sustainable design, and biomechanics.

\section{Awilma Paola Ventura Lugo, Western Michigan University}

Awilma Ventura is a graduate student in manufacturing engineering at Western Michigan University. She comes from the Dominican Republic and did her undergraduate studies in mechanical engineering at Utah State University. 


\section{Virtual instrumentation for study of a fluid power system}

\section{Introduction}

Recognizing the global demand for energy and its impact on the environment, practice of energy efficiency in all products and processes is essential for future sustainable industries. Over $80 \%$ of the energy used worldwide comes from the finite nonrenewable sources and this is supposed to increase significantly in coming years. According to U.S. Energy Information Administration's International Energy Outlook 2017, world's energy consumption is expected to increase by $48 \%$ between year 2015 and 2040.

A fluid power system [1] transforms available form of energy to mechanical energy through the use of a fluid media. It also deals with transmission and control of forces and motion using a pressurized fluid. In general, a fluid power system, specifically hydraulics system is a costeffective solution of problems requiring high power density and large reversible force or torque in an application. It is also easy to control and can be integrated with a mechanical or electrical system in complex applications. As pressurized fluid circulates, hydraulic systems waste much of their energy, regardless of the amount of work output. Most industrial hydraulic applications are not designed with the goal of minimizing their energy consumption [2], therefore, thermodynamically, they tend to be inefficient. Various study [3, 4] investigated methods to incorporate overall efficiency of hydraulic system in design of applications.

In typical hydraulic system, overall efficiency may vary from $6 \%$ to $60 \%$. Literature of energy conservation and efficiency research includes a variety of efforts to analyze hydraulic systems under different types of conditions. Borghi et al. [5] investigated the problem of reducing power dissipation of hydraulic circuits of off-road vehicles. To conserve energy, it used a lumped parameter approach for an alternative hydraulic circuit architecture of agricultural tractors. Kilic et al. [6] presented a study to predict the pressures in the cylinder chambers of a variable-speed pump that was monitored and controlled by using neural network with linear and Kalman filters. Another work [7] by Puddu and Paderi proved that behavior of real gas has a considerable influence on the size of accumulators used for kinetic energy recovery of vehicles.

Thermodynamic properties of gases were analyzed to find the differences between ideal and real gas compressibility. Different gases were tested as charging fluid in a hydro-pneumatic accumulator. The results showed distinctive characteristics in volumetric gas properties, and thermal efficiency of the accumulator. Sola et al. [8] proposed to improve energy efficiency in industrial motor system using a multicriteria analysis. It recommended the dissemination of multicriteria decision aiding to support the decision makers in industries and to improve energy efficiency in system powered by electric motors. Ramakrishnan et al. [9] developed a multiobjective design optimization procedure based on an evolutionary strategy algorithm that maximizes the energy efficiency of a hydraulic system. The study showed that optimizing the system parameters increased the energy efficiency by $3 \%$ and hydraulic regeneration efficiency by $17.3 \%$. Shaoyan et al. proposed efficiency based regenerative braking control strategy for hydraulic auxiliary braking system of vehicle [10]. The braking system had variable speed fluid coupling, clutch, blocking clutch, quantitative axial direction plunger pump/motor, accumulator, and control system. An efficiency curve was developed, and all losses were calculated using 
control system and MatLAB. A conclusion was made that with lower rotating speed and low volumetric efficiency, there would be low holistic efficiency which is not suitable for energy regeneration [10].

Typically, hydraulic system is composed of components and circuit, instrumentation and control system. Connections between all the hoses with respective units along with the flow, temperature, pressure and RPM sensors create the hydraulic circuit of the system where pumps and motors transform the power. The viscosity of a fluid determines the resistance to flow. With higher viscosity, the fluid friction increases, and energy is dissipated as heat. Energy losses occur in various form when there is a change in the cross-section of flow path and direction of the flow in valves, hoses, couplings, filters etc. in a hydraulic system. The more path fluid travels, the greater are the losses. The nature of resistance of valves, hoses and pipes can be determined using experimental methods. A complete analysis of a system requires both analytical and experimental. Losses in pumps are determined by overall efficiency, which is a product of volumetric efficiency and mechanical efficiency of the system. Losses on hoses and other components are comprised of linear losses and can be approached as local losses with relatively small values. Therefore, it is very important to design a hydraulic system with hoses having the appropriate material, shape, and diameter to ensure as less friction as possible. Energy losses in rotary motors are similar to pumps.

In industrial hydraulic system, high-power pumps are designed for low energy consumption, but the low-power pumps are usually focused on low investment costs sacrificing their energy efficiency. Conventional variable speed hydraulic drives exhibit the ruggedness, weight and controllability required for a large system; however, they have acceptable efficiency at full load and efficiency drops as the load changes.

\section{Instrumentation}

Data acquisition tools and programming were needed for the development, implementation, and analysis of the pump system used for experimentation. Digital modules and sensors connected to the parts providing variable input were needed to obtain the required data and analyze the efficiency performance when changing certain parameters. The components of the fluid power system are further described in the prototype development section below.

In order to acquire the data from the experiment, sensors had to be installed to convert physical values into a measurable electrical signal. Then, several NI-DAQ modules were installed as data acquisition measurement hardware that digitizes signals to be interpreted by the computer and LabVIEW was used as the programming tool. The advantages of choosing NI-DAQ and LabVIEW over other data acquisition tools come from their compatibility with each other, processing power, and easy display. LabVIEW software allows to receive the signals from the sensors, show the signals as measurements, easily export the data for analysis, and graphically present results in the desired output. In addition, using Matlab allows to take the data from LabVIEW to identify trends in the overall efficiency performance of the system and test different parameters through statistical analysis of the acquired data and signal processing. In addition, 
Automation Studio is used to simulate the performance of the hydraulic system. The simulation provides an expected efficiency performance and another comparison point for data analysis.

The pressure, temperature, flow, torque, and RPM measurements are collected using National Instruments Data Acquisition (DAQ) hardware. A cDAQ-9188 chassis is used to collect signals from a NI 9207 analog voltage and current sensing module contained in slot 1 in the chassis. The wiring of the NI 9207 module is shown in Figure 1. The positive lead of power supply is wired directly to a Vsup pin, 19, with a 2 A quick burn inline fuse and the ground of the power supply is attached to a common ground pin, 10. The positive pressure transmitter lead is connected to a Vsup pin, 30, and the negative lead is connected to a current sensing channel, AI8. The voltage of the cell is measured via the $\mathrm{AIO}+$ and $\mathrm{AIO}$ - pins. A $1 \mathrm{M} \Omega$ resistor is connected to pin 28, the common ground and has a common node with the negative voltage lead. All the other sensors, i.e., temperature, flow and torque are also connected the same way but to different pins. The sensors are set to collect data at a frequency of $20 \mathrm{~Hz}$.
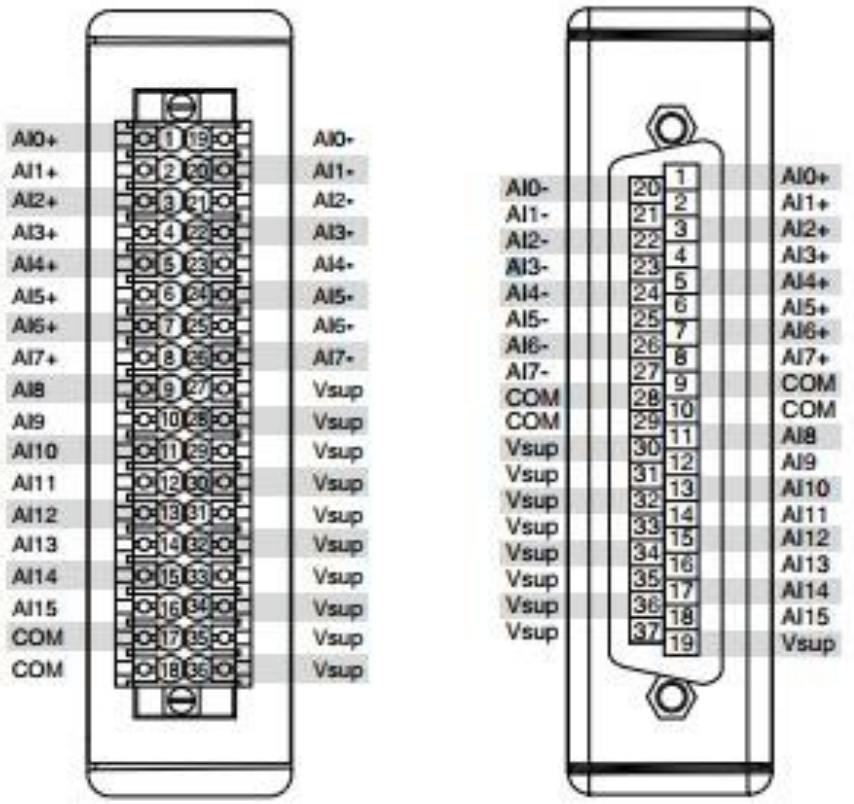

Figure 1: NI 9207 pin out diagram

The torque sensor used is a rotating torque sensor from Omega TQ513-500 series. The sensor measures the torque produced by the fluid power system using shaft-to-shaft in-line placement. In addition, the sensor has a 10- pin twist lock connector that provides the option of connecting an optical encoder to measure angle or speed. The maximum shaft speed is 5000 RPM and torque of $500 \mathrm{in}-\mathrm{lb}$. This torque sensor creates two separate signals, one for torque which requires either a Wheatstone bridge conditioner module such as NI-9237 or an optical encoder for sensing speed. The torque sensor creates a pair of pulse trains that require a counter input module such as the NI9361 or a suitable digital module such as the NI-9401.

Using the graphical programing language of LabVIEW, a Virtual Instrument (VI) is created to collect, display, and record the data from the sensors. The block diagram and VI Front Panel are 
shown in Figure 2 and 3. In separate charts, real time pressure, temperature, flow, and torque are displayed. The VI allows saving the output in a csv file that is used for detailed analysis.

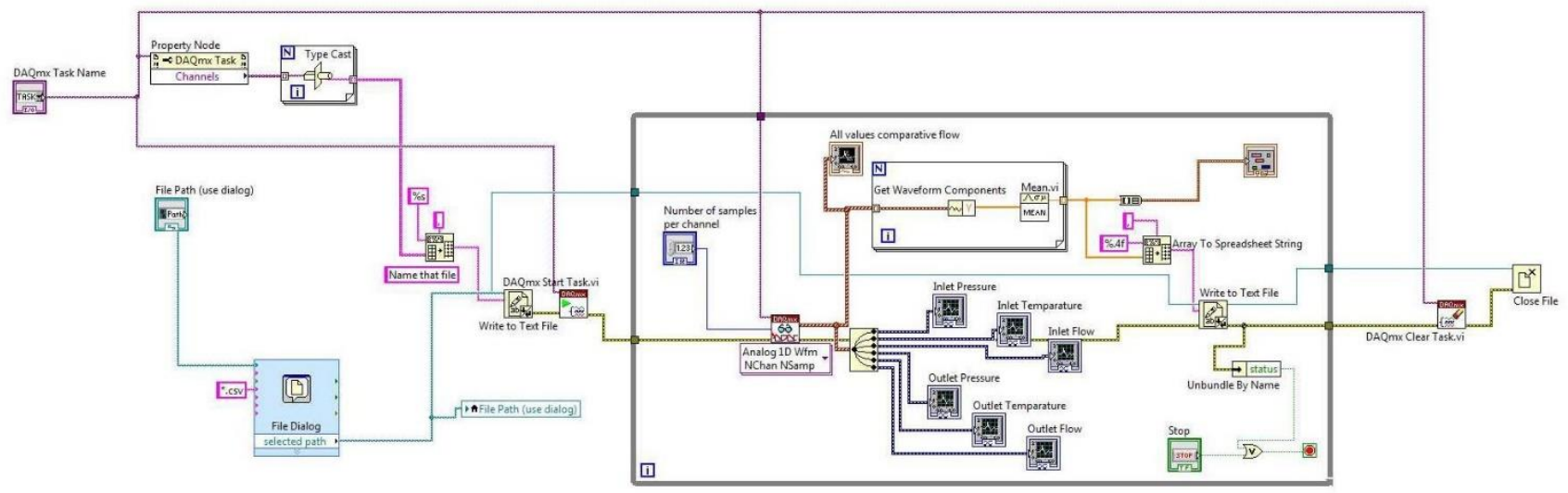

Figure 2: Block Diagram

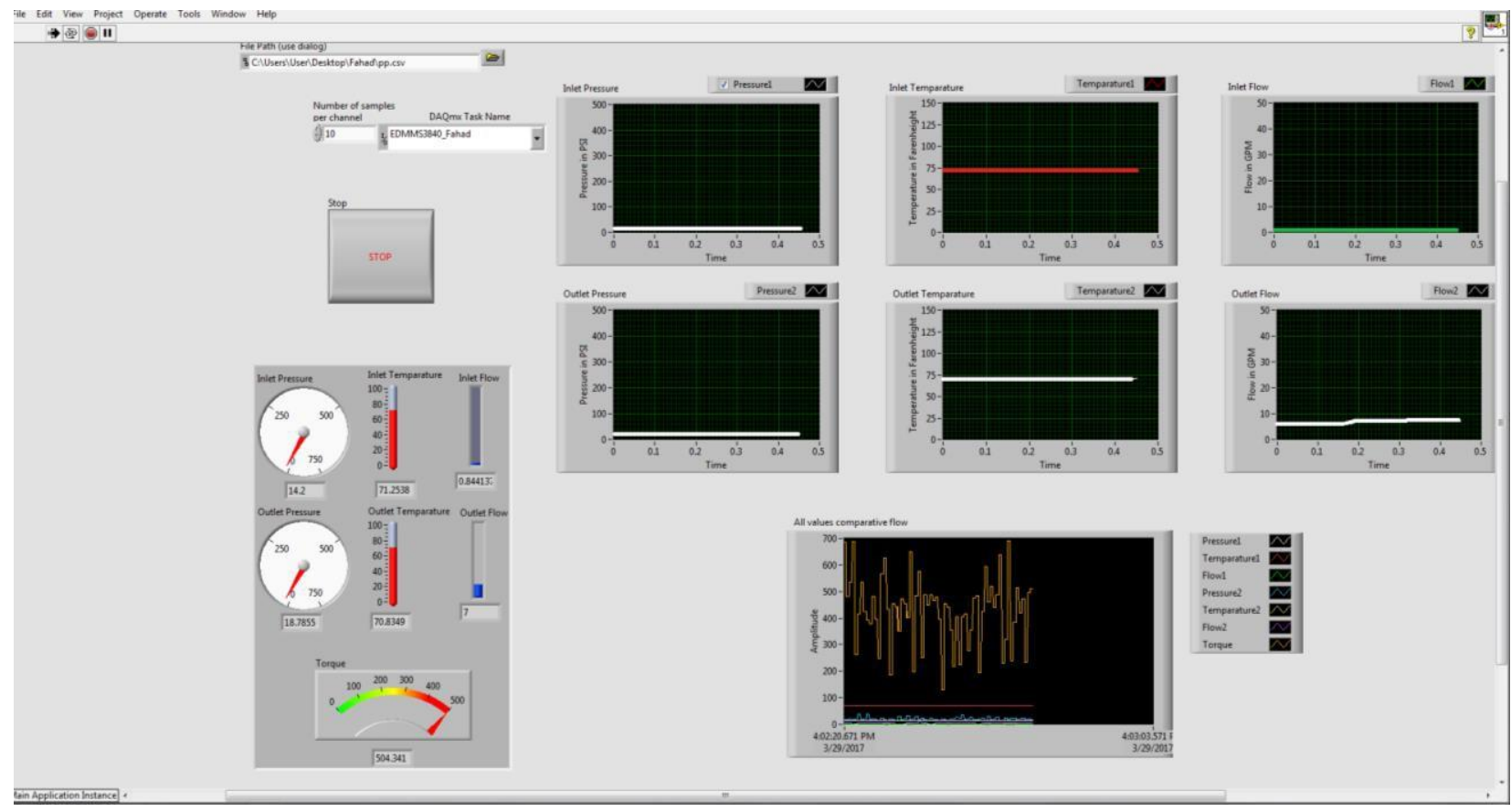

Figure 3: VI front panel

\section{Prototype development}

The prototype of an application involving performance of a small pump is developed in the laboratory using a modular hydraulic system [11]. The power unit of the system consists of an electrically driven pump, reservoir, and supply and return manifolds. The hydraulic system for the study consisted of a small pump and hydraulic motor, flow control valves, relief valves, 
hoses, and fittings. Pressure transducers, flowmeters, thermocouples, and torque/RPM sensor monitor process parameters. Pipes, hoses, and tubes serve as the conductors that distribute fluid between the pump and remaining system components.

To perform the fluid control function in a system, three types of valves are used, namely, directional control valves, pressure control valves, and flow control valves. Directional control valves control where in the flow circuit the fluid will flow. Pressure control valves limit the maximum pressure as well as lower pressure in different parts of a system. Flow control valves controls fluid flow rate to control the rate of movement of an actuator in sections of a system. A fluid conditioning system is used to remove contaminants and maintain proper temperature of the hydraulic fluid. As hydraulic oil moves from the reservoir to pump and runs through a system an additional filter at the return manifold clears impurities prior to return to the tank. The first system component the oil encounters is the pressure control valve, which sets system pressure.

Next comes the directional control valve, that directs the oil to the motor. Oil then moves out into system lines and returns to the reservoir through the directional control valve. Oil from the other side of the piston is returned to the reservoir. A relief valve is located between the pump and the directional control valve to protect the system from potential high-pressure risks. In case of high pressure, some oil will return to the reservoir and ensure pressure does not exit the set limit. A flow control valve restricts the oil flow in ways other than controlling shaft speed and supplies an established volume of oil to the motor. A schematic of the hydraulic system is shown in Figure 4.

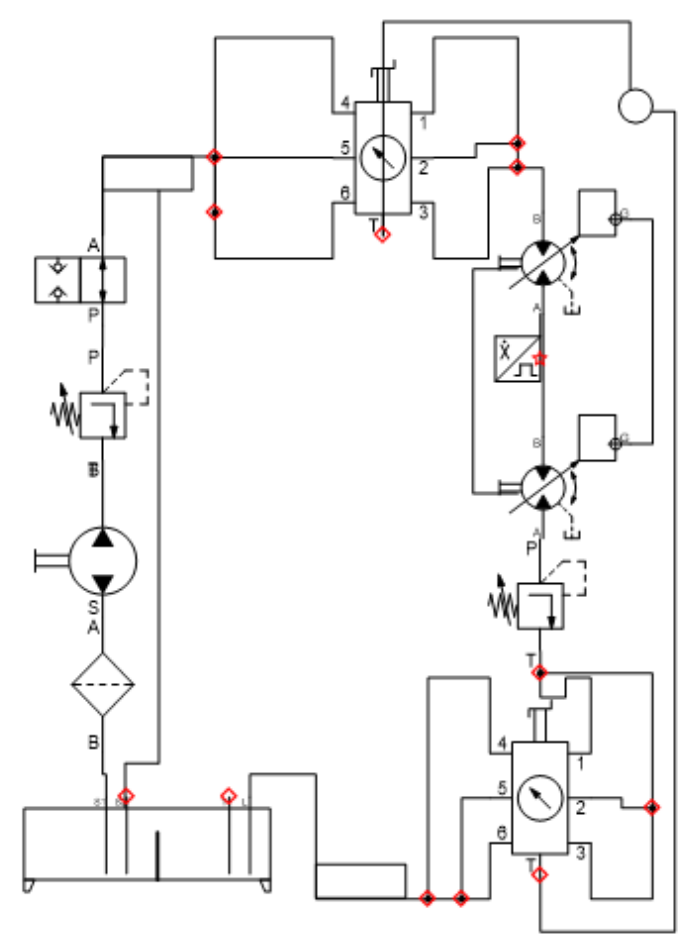

Figure 4: Schematic of the hydraulic system 


\section{Data analysis}

Fluid behavior concepts had to be used to analyze the acquired data. The Navier Stokes equation is used to analyzed fluid flow characteristics in a hydraulic system as shown in equation (1).

$$
\frac{\partial \rho}{\partial t}+\nabla(\rho V)=0
$$

Where $\rho=$ density, $V=$ flow velocity, $\nabla=$ vector divergence operator for a general flow field, and $t=$ time.

The efficiency of the pump can be obtained in terms of the following $P$ output pressure, flow rate $Q$, torque $\tau$, and angular velocity $\omega$ as shown in equation (2).

$$
e=\frac{P Q}{\tau \omega}=F_{1}(P, Q, \tau, \omega) \ldots .
$$

To determine the variables that affect pump efficiency of the fluid system the variables that affect equation (2) were defined. Identifying that flow rate $Q$ is a function of $\mathrm{q}=$ displacement volume, $\omega=$ angular velocity, $\mu=$ fluid viscosity, and $T=$ temperature. In addition, the overall efficiency function shown in equation (3) was determined by knowing that output pressure $\mathrm{P}$ is a function of $\tau=$ torque, $Q=$ flow rate, $\mu=$ fluid viscosity, and $\mathrm{T}=$ temperature.

$$
e=F(q, \omega, P, Q, \tau, \mu, T) \ldots(3)
$$

In general, the efficiency of any pump system is a function of many independent variables and the parameters used to control the variables in the process. The objective of this study is to optimize the efficiency of a pump by controlling the operational parameters of the process in equation (3) and determining which have the most impact in the overall efficiency. In addition, the efficiency function of the pump is complex and nonlinear in nature, meaning that the numerical analysis of this type of function can be a difficult task.

To determine the impact of each variable affecting the efficiency function in equation (3), a sensitivity analysis can be used to examine the relationship between variable parameters of the fluid power system [12]. Prior to optimization of the efficiency, relatively more sensitive process parameters are identified. In the optimization process, most of the efficiency gain in the pump operation are achieved through controlling these parameters.

The experimental data was collected from the setup shown in Figure 4. The data acquisition module collected and recorded data at 20 points in every second. After getting torque, RPM, Flow and Pressure data, efficiency was calculated. Figure 4 illustrates the efficiency of the hydraulic system when a 2 HP pump is used. The efficiency of the system was then calculated. At 660 RPM, system's overall efficiency is $74.07 \%$. As RPM increases, system efficiency also increases. At 1686.36 RPM, system efficiency is maximum, $83.14 \%$ which is very close to the known theoretical efficiency of $85.5 \%$ of the pump. After efficiency of the system reaches the 
highest point, a drop in the efficiency was noticed with increase in RPM. This is the expected result for a hydraulic pump. The efficiency is not as exact as the theoretical one which can be of several issues. Presence of foreign particle on the system, overheating of oil, increase in system leakage etc. can cause efficiency to be lower than expected.

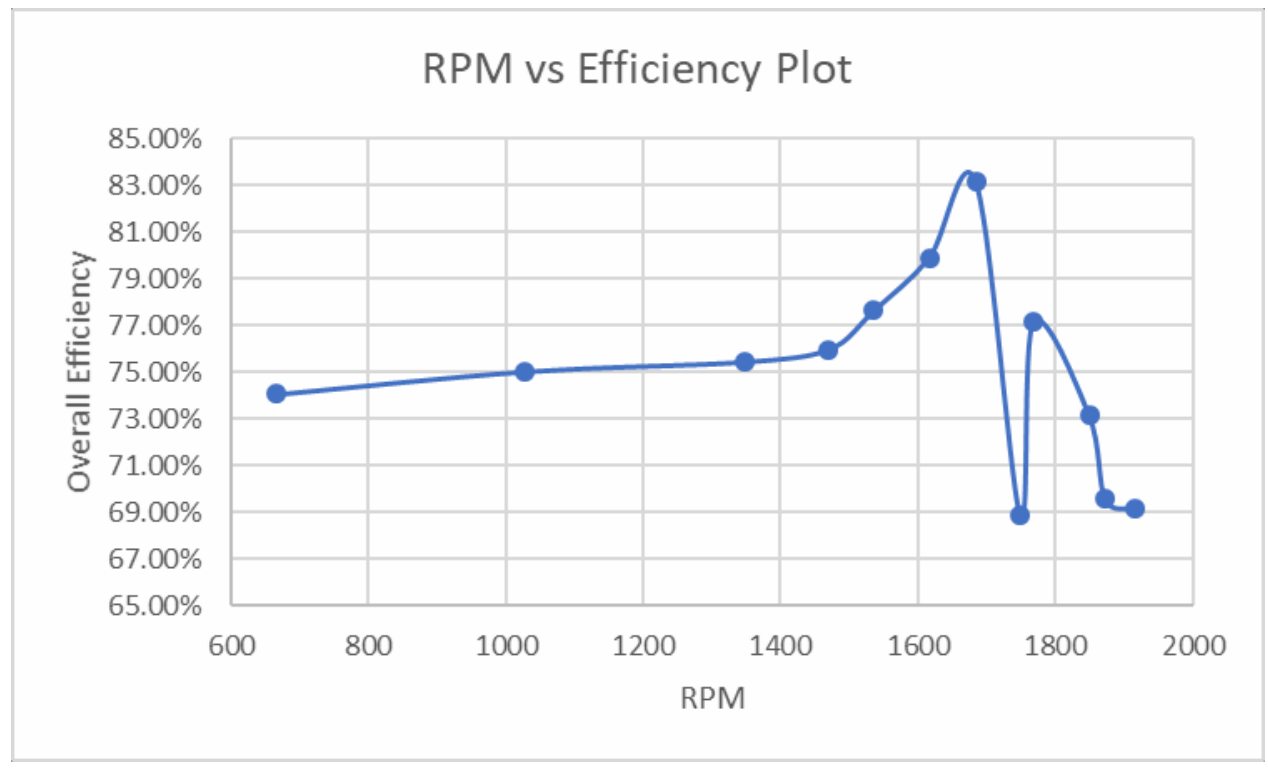

Figure 4: Efficiency vs RPM Plot

The efficiency calculation mentioned above is based on RPM and Pressure data only. The effects of temperature, viscosity, and other variables were not taken into consideration. The next phase for the study is to gather more data and calculate the efficiency more accurately. Further analysis will provide with necessary information and procedures for optimization of the system. The pump system must be capable of delivering the flow at a maximum efficiency. Pump operates at a particular viscosity fluid at given temperatures and pressures. With increase in temperature, a reduction in viscosity or an increase in operating pressure occur, which causes more leakage across the pump and consequently reduces the pump efficiency. As the pump wears, the leakage will increase. Upon identifying factors that affect the overall efficiency, the next step is to establish which factors play the most vital role. Determining the best possible settings for these crucial factors that result in the optimum value of overall efficiency is the goal. To analyze the system, a model can be created where pressure, temperature and flow in inlet and outlet, RPM and Torque will be selected as parameters and system efficiency will be selected as output. The sensitivity analysis will find the most significant parameters for optimizing efficiency. According to this result, further analysis will be done using Excel or Minitab considering only the crucial parameters. This will give us exact pressure, temperature, torque or RPM at which, the system efficiency will be maximum. The next step is to verify this result by running the system at those parameter values. 
Currently, the different types of valves in the system are manually controlled. Therefore, to reduce human error from manual control and change the critical parameters for efficiency, an automated control system as Programmable Logic Controller (PLC) will be introduced in the next step of this research. The PLC system in development is shown in Figure 5.

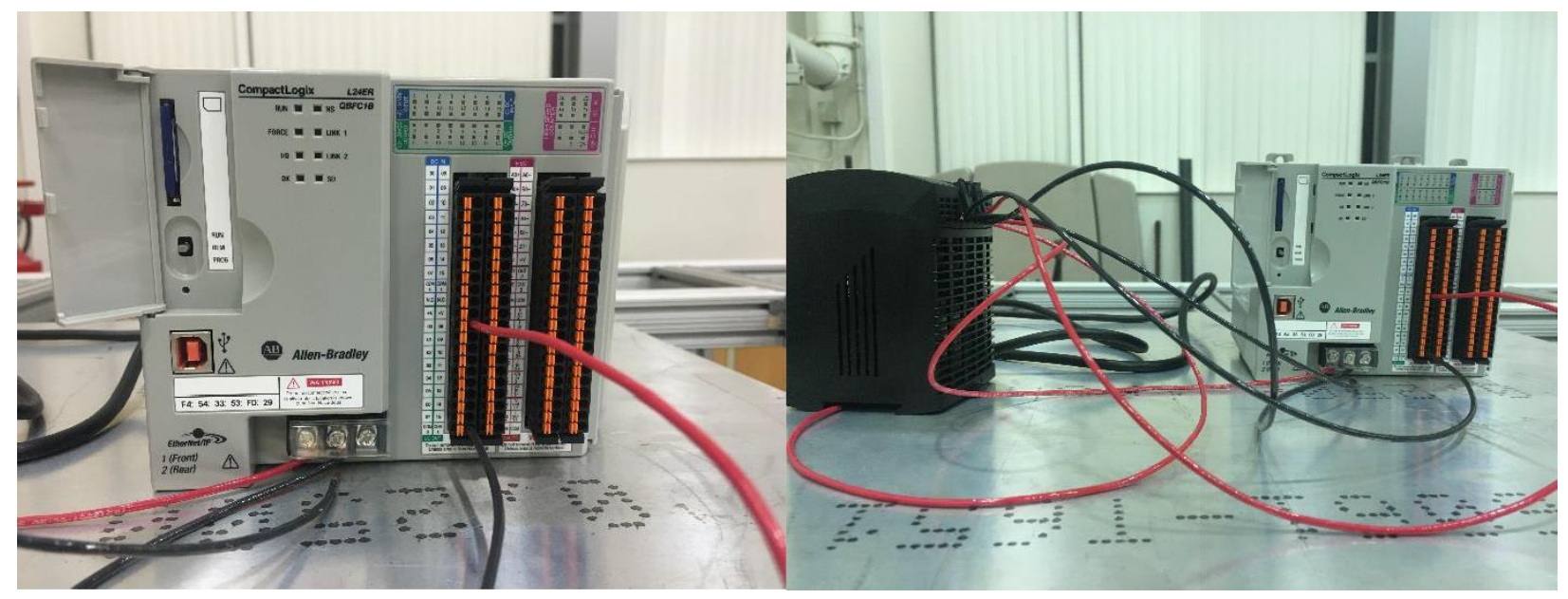

Figure 5: CompactLogix Allen-Bradley PLC Controller

The implementation of the PLC controller will allow to manipulate the different valves used in the system through computer programming, which reduces manual handlining in the system. The goal of the PLC program will be to set, monitor, and control the needed parameters for optimal efficiency with a computer program.

\section{Conclusion}

A prototype of a hydraulic system was developed and modified in the laboratory by adding a new sensor to measure torque and RPM of the system and control parameters impacting the overall efficiency. The performance data of the pump was acquired through pressure, temperature, flow rate, and torque sensors. The relationship between the overall efficiency of the pump and variable parameters is established through a sensitivity analysis. The collected data shows that output pressure $\mathrm{P}$ and shaft speed are the ones with most impact on efficiency. Monitoring and controlling the major parameters can be used to optimize the overall efficiency of the system, which at an industry level would reduce cost and produce more earnings. The experimentation allowed a greater understanding on pump efficiency. Finally, a PLC control system as will be implemented for more detailed investigation of the problem and eventually use the study to improve energy efficiency of a hydraulic system. 


\section{References}

1. Sullivan, J., Fluid Power Theory and Applications, Prentice Hall Inc., Upper Saddle River, New Jersey, 1998.

2. Rydberg, K.; Energy Efficient Hydraulics - System solutions for loss minimization; National Conference on Fluid Power, Linkoping University, Sweden. March 2015.

3. Choudhury, A. and Rodriguez, J.; Experimental Analysis for Energy-efficient Product Design, Journal of Engineering Technology, Volume 34(1), 2017.

4. Choudhury, A., Rodriguez, P. Ikonomov, J. He, B. De Young, R. Kamm, S. Hinton, Human powered energy efficient vehicle design, Proceedings the American Society for Engineering Education Annual Conference, San Antonio, TX, June 2012.

5. Borghi, M., Zardin, B. Pintore, F., and Belluzi, F.; Energy Savings in the Hydraulic Circuit of Agricultural Tractors, $68^{\text {th }}$ Conference of the Italian Thermal Machines Engineering Association, Energy Proceedia 45, 2014.

6. Kilic, E., Dolen, M., Caliskan, H., Koku, A. B., \& Balkan, T.; Pressure prediction on a variable- speed pump controlled hydraulic system using structured recurrent neural networks. Control Engineering Practice, Vol. 26, pp 51-71, 2014.

7. Puddu, P., \& Paderi, M.; Hydro-pneumatic accumulators for vehicles kinetic energy storage: Influence of gas compressibility and thermal losses on storage capability, Energy, Vol. 57, pp 326-335, 2013.

8. Sola, A., Mota, C., and Kovaleski, J.; A model for improving energy efficiency in industrial motor system using multicriteria analysis. Energy Policy, Vol 39(6), 2011.

9. Ramakrishnan, R., Hiremath, S., and Singaperumal, M.; Design strategy for improving the energy efficiency in series hydraulic/electric synergy system. Energy, Vol. 67, pp. 422-434, 2014.

10. Shayoan, G., Fei, T., Bin, S., Jiangang, L., \& Yao, S. (n.d.). Research on Efficiency Optimization for Hydrostatic and Hydraulic Auxiliary Braking System of Tracked Vehicle. Retrieved from https://ieeexplore-ieee. org.libproxy.library.wmich.edu/document/6154269/citations?tabFilter=papers\#citations.

11. Choudhury, A. and Rodriguez, J.; A Modular System for Energy Efficiency Study of Hydraulic Applications, Proceedings of the ASEE Annual Conference, New Orleans, LA, June 2016.

12. Saltelli A., Ratto M., Andres T., Campolongo F., Cariboni J.,Gatelli D., Saisana M., and Tarantola S.,Global Sensiti-vity Analysis: The Primer, John Wiley and Sons, Ltd., 2008. 